\title{
Sector-Based Assessment of Infarct Size on Late-Gadolinium-Enhancement MRI in a Mouse Model of Acute Myocardial Infarction
}

\author{
Cheongsoo PARK, ${ }^{1,2} \mathrm{MS}$, Eun-Hye PARK, ${ }^{2} \mathrm{MS}$, Kiyuk Chang, ${ }^{2} \mathrm{MD}$, \\ and Kwan Soo Hong, ${ }^{1} \mathrm{PhD}$
}

\begin{abstract}
SUMMARY
Scoring of myocardial infarction (MI) disease extent in cardiac magnetic resonance (CMR) images has been generally presented in terms of area-based infarct size. However, gradual thinning of the infarcted wall and compensatory hypertrophy of the noninfarcted remote wall during left ventricular (LV) remodeling after MI complicate the accuracy of infarct size measurement. In this study, we measured and compared infarct sizes in mice on late gadolinium enhancement (LGE) images using area-, length-, and radial sector-based methods.

MI was induced by permanent ligation of the left coronary artery $(n=6)$. LGE images were acquired 30 minutes after intravenous injection of Gd-DTPA-BMA. Percentages of infarct size (\%Area, \%Length, and \%Sector) on the LGE images were calculated and compared with histological findings.

Infarct sizes obtained by an area-based approach were smaller than those obtained by other measurements. The area-based approach underestimated infarct size compared with the length-based approach. Most infarct sizes measured by each method demonstrated a similar trend, with maximum values determined by sector-based measurements using a mean + SD threshold. Spearman's rank correlation coefficients indicated that the 3 measurements were strongly correlated $(P<0.05)$ to each other. Significant differences and trends were observed between sector-based infarct sizes with different thresholds when 16 or more sectors were used.

In conclusion, our study demonstrated that methods used for the histological calculation of infarct size could be applied to CMR analysis. Moreover, our results showed a similar trend to histological assessment. Sector-based CMR approaches can be useful for infarct size measurement. (Int Heart J 2016; 57: 736-741)
\end{abstract}

Key words: Cardiac magnetic resonance, Coronary artery permanent ligation, Infarct measurement

$\mathrm{C}$ ardiac magnetic resonance (CMR) markers of myocardial damage at the time of acute myocardial infarction (MI) including infarct size provide independent and incremental prognostic information in addition to clinical risk scores and left ventricular (LV) ejection fraction. ${ }^{1)}$ Multiple lines of evidence have demonstrated that measurements of the extent of myocardial injury, such as larger infarct size and reduced LV ejection fraction, are closely associated with longterm cardiovascular morbidity and mortality. ${ }^{2,3)}$ Accordingly, the accurate measurement of infarct size is crucial for determining the prognosis of patients.

CMR has been established as a gold standard for the assessment of cardiac structure and function because of its excellent soft tissue contrast, high spatial resolution, and reproducibility, ${ }^{4-6)}$ which enables the comprehensive evaluation of markers of myocardial damage in patients with acute MI. Inf- arct size is assessed using late gadolinium enhancement (LGE) short-axis images covering the entire LV, because LGE is the most sensitive imaging technique to detect injured myocardial tissue, which has enhanced permeability to the extracellular gadolinium chelate contrast agent. ${ }^{7.8}$

Infarct size can be measured by several methods ${ }^{9,10)}: 1$ ) area-based measurement, mainly used in clinics, is calculated by dividing the infarct area by the area of LV myocardium; 2) the length-based approach uses the length of infarcted and full circumferential LV wall; and 3) angle-based calculation involves measurement of the angle between border zones of the infarct region from the centroid of the LV lumen and dividing by $360^{\circ}$.

The area-based approach is the most popular measurement for the estimation of infarct size. ${ }^{11,12)}$ However, because of thinning of infarcted LV wall and hypertrophy of remote vi-

From the ${ }^{1}$ Bio-imaging Research Team, Korea Basic Science Institute, Cheongju and ${ }^{2}$ Cardiovascular Center and Division of Cardiovascular Medicine, Seoul St. Mary's Hospital and College of Medicine, The Catholic University of Korea, Seoul, South Korea.

This study was supported by a Creative Allied Project (CAP) grant (PBF050, KSH) funded by the Korea Research Council of Science and Technology (KRCF), a KBSI grant (D35401, KSH), and a Sanofi-Aventis Korea grant (KC).

Addresses for correspondence: Kwan Soo Hong, PhD, Bio-imaging Research Team, Korea Basic Science Institute, MRI Bldg, 162 Yongudanji-ro, Ochang, Cheongwon-gu, Cheongju 28119, South Korea. E-mail: kshong@kbsi.re.kr or Kiyuk Chang, MD, Cardiovascular Center and Division of Cardiovascular Medicine, Seoul St. Mary's Hospital and College of Medicine, The Catholic University of Korea, 222 Banpo-daero, Seocho-gu, Seoul 06591, South Korea. E-mail: kiyuk@ catholic.ac.kr Received for publication February 1, 2016. Revised and accepted April 20, 2016

Released in advance online on J-STAGE November 4, 2016.

All rights reserved by the International Heart Journal Association. 
able myocardium after MI, the area-based calculation has intrinsic limitations for the accurate measurement of infarct size. Although length- and angle-based approaches are simpler and more reliable for the calculation of infarct size compared to area-based estimation, the measurement of infarct length and angle is operator-dependent, which limits their widespread clinical application. There are ambiguities in the measurements of infarct length and angle in situations of subendocardial or subepicardial enhancement. Furthermore, the determination of the optimal centroid for infarct angle measurement is somewhat vague and inconvenient. Compared to these techniques, the sector-based approach only requires endocardial and epicardial segmentation. The LV lumen centroid can be calculated from endocardial or epicardial boundaries.

We hypothesized that the sector-based approach is a simple and reliable method that could provide similar results to length-based measurement, which is less affected by morphological changes. ${ }^{9,10)}$ We estimated infarct size on LGE images using area-, length-, and radial sector-based methods, and correlated the estimated infarct sizes obtained by these 3 methods with histological measurements in the acute stage of MI in mice.

\section{MeTHODS}

Animal model: All animal procedures were approved by the Institutional Animal Care and Use Committee of the Korea Basic Science Institute. Six male C57BL/6 mice ranging from 10 to 14 weeks of age were used in this study. Mice were anesthetized by inhalation of a mixture of isoflurane $(1.5 \%)$ and oxygen. After anesthesia, mice were intubated with an endotracheal catheter and ventilated using a rodent ventilator (Harvard Apparatus, Inc., USA) and placed on a temperature-controlled heating pad. A left thoracotomy through the fourth intercostal space was performed. The pericardial sac was opened to access the heart, and permanent ligation of the anterior descending branch of the left coronary artery was achieved by tying 8-0 nylon suture around the artery. Myocardial ischemia was confirmed by observing blanching and dyskinesis of the anteroapical region of the left ventricle (LV) distal to the suture.

Magnetic resonance imaging: All MRI experiments were performed on a 4.7 T animal MRI system (Biospec 47/40, Bruker BioSpin, Germany). One day after MI surgery, CMR was performed while the mice were anesthetized with $1.5 \%$ isoflurane in oxygen. The RF coil to transmit pulses and receive signals was a 35-mm-diameter birdcage resonator. Mouse body temperature was maintained at $\sim 37^{\circ} \mathrm{C}$ using a warm air blower. Electrocardiography (ECG) electrodes were inserted into the fore and hind limbs, and a respiration pillow was taped across the chest. ECG and respiration signals were monitored using a small-animal monitoring unit (SA Instruments, Inc., USA). Short-axis CMR image acquisition was both ECG-gated and respiratory-gated. LGE CMR images were acquired $30 \mathrm{~min}$ utes after intravenous injection of $0.3 \mathrm{mmol} / \mathrm{kg}$ Gd-DTPABMA (Omniscan ${ }^{\circledR}$, GE Healthcare, USA). For $\mathrm{T}_{1}$-weighted FLASH imaging, sequence parameters were as follows: TR/ $\mathrm{TE}=73 / 2.7 \mathrm{~ms}$, flip angle $=60^{\circ}$, field of view $=30 \times 30 \mathrm{~mm}^{2}$, matrix size $=256 \times 256$, and slice thickness $=1 \mathrm{~mm}$.

Histology: After all CMR experiments, mouse hearts were extracted on day 1 post-MI for histological evaluation. Extracted hearts were washed with saline, embedded in optimal cutting temperature compound (Sakura Finetechnical, USA), and frozen. Using a cryotome, hearts were cut into 5- $\mu \mathrm{m}$ sections and stained with hematoxylin and eosin (H\&E) and Masson's trichrome (Sigma-Aldrich, USA) according to the protocol provided by the manufacturer. Specimens were evaluated on an Olympus SZX16 (Olympus Optical, Japan) microscope and captured digitally with a charge-coupled camera.

MRI image analysis: All CMR image analyses were performed using freely available software, Segment (Medviso, Sweden) for area-based and sector-based analyses and OsiriX (Pixmeo, Switzerland) for length-based analysis. LV endocardial and epicardial borders at the mid-ventricular level were manually delineated. For area-based analysis, the hyperintense infarcted myocardium was traced manually on the LGE CMR images. Percentage of infarct area (\%Area) was calculated by dividing the infarct area by the total LV area. For the lengthbased approach, endocardial and epicardial circumferences of the LV and the midline of the infarcted myocardium that included $>50 \%$ of the myocardial thickness were drawn. LV circumference measurements were obtained by calculating the mean of the LV endocardial and epicardial lengths. Percentage of infarct length (\%Length) was defined as the infarct midline length divided by the entire LV length and multiplying by 100 . Radial sector values were obtained from the region of the LV, segmented into $8,16,32,180$, or 360 radial sectors, ie, the angular resolutions ranged from $1^{\circ}$ to $45^{\circ}$. Infarct size derived from the sector-based approach (\%Sector) used a mean value and a standard deviation (SD) of the remote noninfarcted LV wall. Three different threshold values were specified; mean + $\mathrm{SD}$, mean $+2 \mathrm{SD}$, and mean $+3 \mathrm{SD}$. Infarct size was calculated as follows: [(the number of sectors above the thresholds) / (the number of total radial sectors) $] \times 100$.

Histology image analysis: Masson's trichrome-stained sections were analyzed with Image $(1.47 \mathrm{v}$; NIH, USA) to measure infarct size. The percentage of infarcted myocardium was calculated using both area-based and length-based measurements as described in the MRI image analysis section. With the area-based approach, infarct size was determined as a ratio of the infarcted myocardial area to total LV area. For lengthbased infarct size measurement, the infarct length with $>50 \%$ transmural extent of myocardial infarction was divided by the perimeter of the $\mathrm{LV}$, and the mean of epicardial and endocardial length.

Statistical analysis: Statistical analysis was performed using OriginPro 9.1 (OriginLab, UK). For all analyses, a value of $P$ $<0.05$ was considered statistically significant. Friedman's ANOVA analysis was used to test the null hypothesis that the infarct sizes determined by \%Area, \%Length, and \%Sector $($ mean $+\mathrm{SD}$, mean $+2 \mathrm{SD}$, and mean $+3 \mathrm{SD})$ were not statistically different. An overall significance value $(P<0.05)$ was found for rejecting this null hypothesis. Accordingly, pairwise differences between each pair among the 4 groups were assessed using Dunn's multiple comparisons test. Spearman's rank correlation coefficient was calculated to assess relationships between the infarct size measurements. Agreement of infarct size between each method was evaluated by means of the Bland-Altman method, and limits of agreement were calculated. Bias was defined as the mean difference between the two methods. The limits of agreement were defined as the $95 \%$ confidence interval of the mean difference between the two 

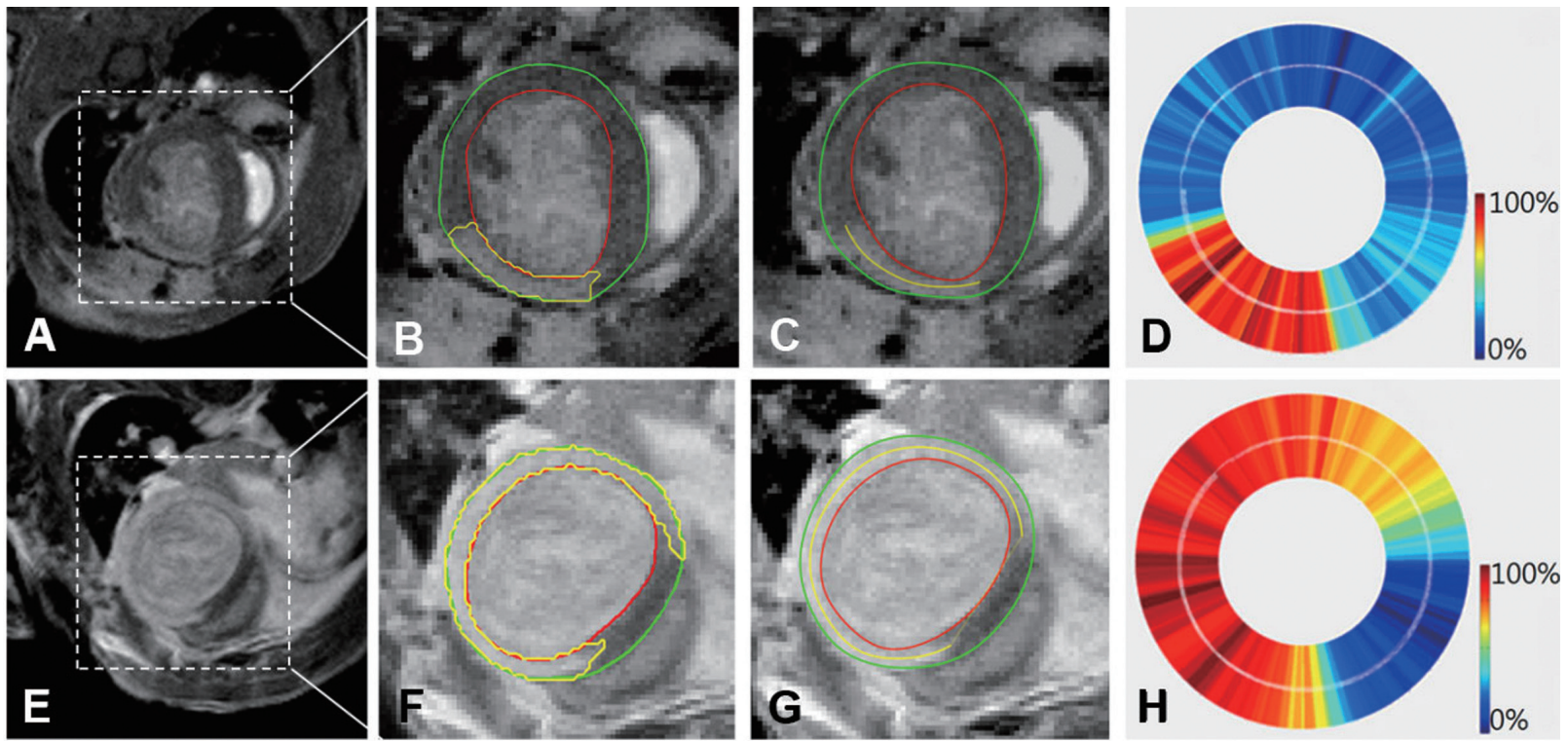

Figure 1. Representative cardiac magnetic resonance images with the area-based $(\mathbf{B}, \mathbf{F})$ and length-based $(\mathbf{C}, \mathbf{G})$ regions of interest and the polar plots of the radial sector-based analysis $(\mathbf{D}, \mathbf{H})$. Endocardial and epicardial borders are outlined in red and green colors, respectively. Infarct area and length are delineated by yellow lines in $\mathbf{B}, \mathbf{C}, \mathbf{F}$, and $\mathbf{G}$. Radial plot of relative signal intensity distribution is shown on a color scale in $\mathbf{D}$ and $\mathbf{H}$.

methods. Page's L trend test was performed to compare and determine the significance of trends in sector-based infarct size determined with different thresholds (mean, mean $+\mathrm{SD}$, mean $+2 \mathrm{SD}$, mean $+3 \mathrm{SD})$.

\section{RESUlTS}

Estimation of infarct sizes with LGE CMR images: Myocardial infarction was successfully induced in all mice by permanent ligation of the LAD, and all infarct regions were clearly observable on LGE CMR images. The infarct size on LGE CMR images was estimated by 3 measurement approaches as described in the Materials and Methods section. Hyperintense regions indicating infarcted myocardium appeared on images in all mice and corresponded to the LAD coronary artery territory. Representative LGE images obtained from two mice with different extents of necrosis and their demarcation using the 3 infarct-size measurements are presented in Figure 1. Top panels show the moderate case with anterior wall injury, and bottom panels the severe case. Mid short-axis images in Figure $1 \mathrm{~A}$ and $1 \mathrm{E}$ show transmural regions of hyper-enhancement in anterolateral and entire LV free wall. Three geometrical delineations for each image by area-based (Figure 1B and 1F), length-based (Figure 1C and 1G), and sector-based (Figure 1D and $1 \mathrm{H})$ approaches are presented. Infarct sizes determined by area-based calculation were $23.7 \%$ (Figure 1B) and $70.1 \%$ (Figure 1F), respectively, compared with $24.9 \%$ (Figure 1C) and $79.3 \%$ (Figure $1 \mathrm{G}$ ) by the length-based approach and $25.8 \%$ (Figure 1D) and $83.3 \%$ (Figure $1 \mathrm{H}$ ) by the sector-based measurement using a threshold of mean +2 SD. Quantitative results of infarct sizes obtained in 6 mice ( 2 slices each) using the 3 approaches are shown in Figure 2. Most infarct sizes measured by each method demonstrated a similar trend, with maximum values determined by sector-based measurement us-

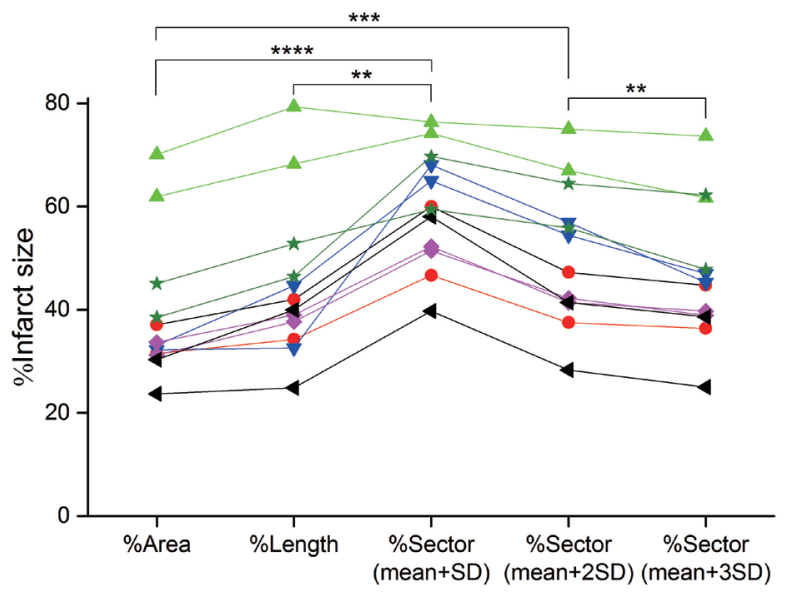

Figure 2. Comparison of infarct sizes derived from the area- (\%Area), length- (\%Length), and sector-based measurements (\%Sector, threshold values: mean $+\mathrm{SD}$, mean $+2 \mathrm{SD}$, and mean $+3 \mathrm{SD}$ ). Infarct sizes obtained by the sector-based approach with mean $+\mathrm{SD}$ threshold were larger than those obtained by other measurements. Each symbol corresponds to infarct sizes measured at the midventricular level on the same images. The values were derived from 6 mice, two slices each. ${ }^{* *} P<0.01 ;{ }^{* * *} P<0.001$; $P<0.0001$

ing a mean + SD threshold $(60.1 \pm 11.1 \%)$. Comparison between area-based and length-based approaches revealed no statistically significant difference $(P=0.2014)$. Infarct size determined by length-based calculation was not significantly different from that determined by sector-based approach with mean + 2SD $(P=0.5281)$ and mean +3 SD $(P>0.9999)$ thresholds, but was significantly different from the sectorbased approach with mean $+\mathrm{SD}$ threshold $(P=0.0167)$. There was no statistically significant difference between the areabased approach and the sector-based approach with mean + 

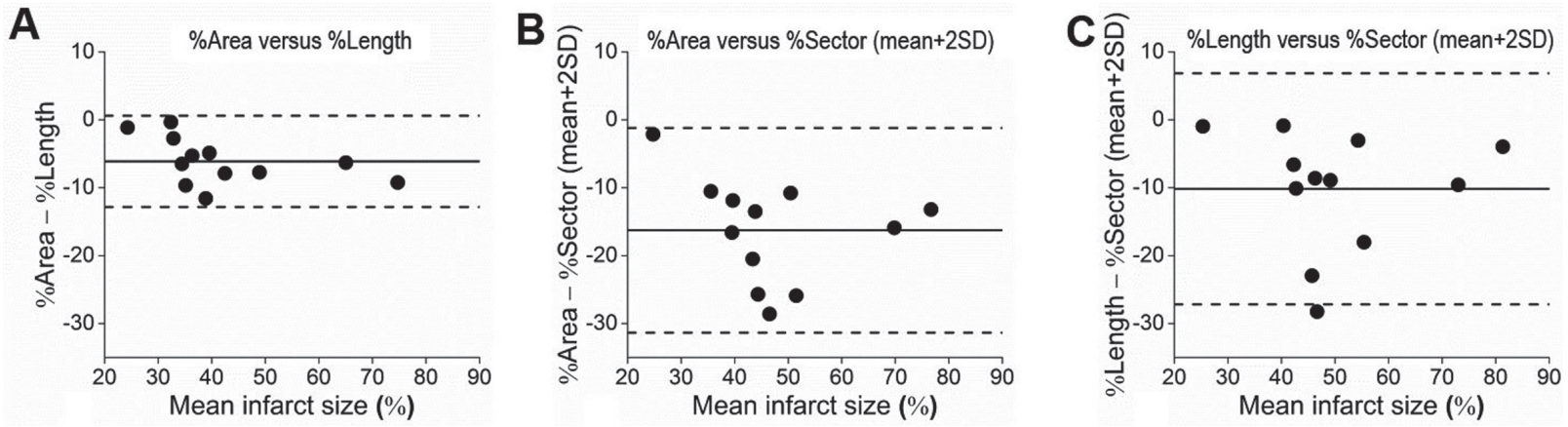

Figure 3. Bland-Altman plots of the 3 measurement approaches showing the differences between each method. Infarct sizes are plotted against the mean infarct size. The solid line denotes bias (mean difference between the measurements), and dashed lines show 95\% limits of agreement (bias $\pm 1.96 \mathrm{SD}$ ).

Table. Analysis of Differences (Friedman test) and Trends (Page's L test) for Infarct Size Within the Six Groups With Different Sector Sizes

\begin{tabular}{cccccc}
\hline & 8 sectors & 16 sectors & 32 sectors & 80 sectors & 360 sectors \\
\hline Friedman test & $P=0.2692$ & $P<0.0001$ & $P<0.0001$ & $P<0.0001$ & $P<0.0001$ \\
Page's L test & $P>0.05$ & $P<0.01$ & $P<0.001$ & $P<0.001$ & $P<0.0001$ \\
(L statistic) & $(148.5)$ & $(159)$ & $(162)$ & $(162)$ & $P<0.001$ \\
\hline
\end{tabular}

3SD threshold $(P=0.2014)$ except for the mean $+\mathrm{SD}(P<$ $0.0001)$ and the mean $+2 \mathrm{SD}(P=0.0002)$ thresholds.

Correlation between 3 measurement methods: Spearman's rank correlation coefficients among the 3 measurement results were strongly positively correlated $(P<0.05)$ to each other, but there was no significant correlation between infarct sizes calculated as \%Length and \%Sector (mean + 2SD) $(\rho=0.515$, $P=0.128$ ). Bland-Altman concordance analysis of infarct size determined by the 3 methods revealed bias and $95 \%$ limit of agreement (Figure 3). All data points were within the $95 \%$ limit of agreement between methods except for one outlier (Figure 3C). Infarct size comparison between \%Area and $\%$ Length (Figure 3A) resulted in smaller bias and narrower variation than the other measurement comparisons (Figure 3B and 3C). Rates of bias between methods were $-5.8 \%$ (\%Area versus \%Length), $-15.9 \%$ (\%Area versus \%Sector [mean + $2 \mathrm{SD}]$ ), and $-10.1 \%$ (\%Length versus \%Sector [mean $+2 \mathrm{SD}]$ ), and corresponding confidence interval ranges between methods were $-5.8 \pm 7.2 \%,-9.7 \pm 8.3 \%$, and $-3.9 \pm 9.3 \%$, respectively (Figure 3 ). A significant difference and trend were observed between sector-based infarct sizes with different thresholds when 16 or more sectors were used (Table).

Histological evaluation: After CMR experiments, mouse hearts were extracted, cryo-sectioned, and stained with $\mathrm{H} \& \mathrm{E}$ and Masson's trichrome for the histological estimation of infarct area. Infarct region on histological sections at the midventricular level was demarcated and measured using ImageJ with both area-based and length-based approaches. A typical LGE image and histological section stained by Masson's trichrome, with the corresponding infarct size measurements, are shown in Figure 4. Infarct region in the subendocardial layer of the inferolateral LV wall was consistently observed in both images (Figures 4A and 4E). Area-based measurement underestimated infarct size compared with the length-based method for both LGE and histology (Figure 4F). Circumferential distribution of signal intensity with the sector-based approach is shown in Figure 4G. Infarct size quantified at the 3 thresholds was
$74.2 \%($ mean $+\mathrm{SD}), 66.9 \%($ mean $+2 \mathrm{SD})$, and $61.7 \%$ (mean $+3 \mathrm{SD})$.

\section{Discussion}

The aim of the present study was to compare 3 different methods to calculate infarct size obtained by CMR imaging in the mouse MI model. We found that the infarct sizes measured by the 3 different measurement methods showed excellent correlations with each other. No significant differences were found between area-based and length-based approaches. Moreover, we observed that the sector-based approach using a minimum threshold of mean $+2 \mathrm{SD}$ could generate similar results to infarct size calculated by the length-based approach. As reported by Takagawa, et $a l^{9)}$ the length-based approach is less affected by morphological changes to the myocardium. They showed that the area-based calculation is more compressed, and therefore the length-based approach is more suitable to calculate infarct size. Our results comparing infarct size measured by the 3 methods indicate that sector-based calculation is also a simple and robust method to assess infarct size.

Since infarct size is the most critical prognostic factor following MI, its accurate measurement is an important indicator of therapeutic efficacy for reducing infarct size in cardiovascular research and preclinical trials. After MI onset, the heart undergoes gradual morphological changes to ventricular geometry such as thinning and shrinkage of the infarcted myocardium and hypertrophy of the remote viable myocardium. However, infarct size is typically based on area measurement of the infarcted region. These morphological changes could affect the calculation of infarct size by the area-based approach and misestimate the real severity of MI. Virag and Murry ${ }^{13)}$ found that infarct size assessed by the area-based approach was reduced from $38 \pm 5 \%$ at 4 days to $20 \pm 4 \%$ at 4 weeks after the onset of MI in a mouse MI model. Lutgens, et $a l^{14)}$ showed that infarct size assessed by the length-based approach was 

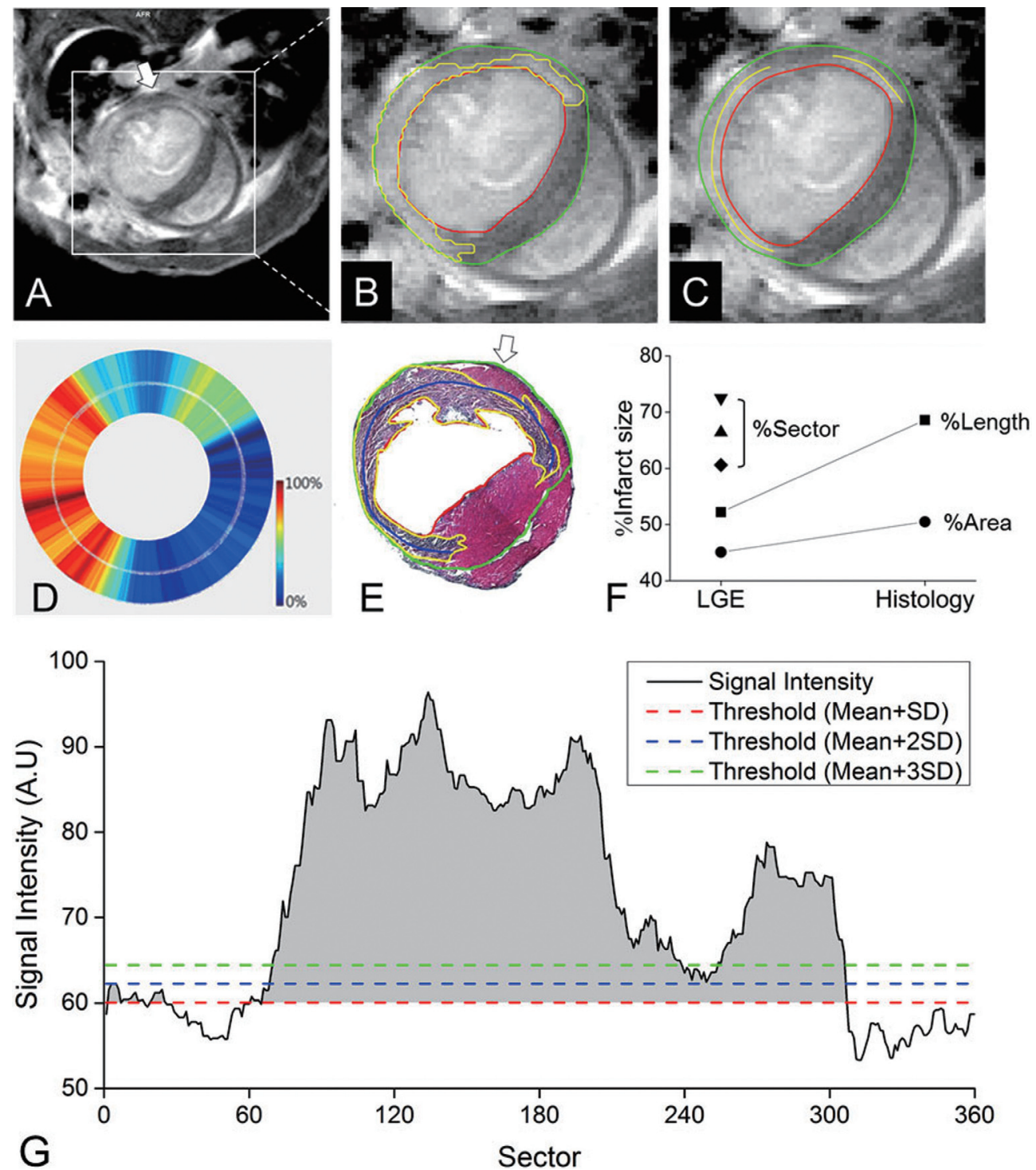

Figure 4. Representative late gadolinium enhancement cardiac magnetic resonance image (A); analysis of infarct size by area-based (B), length-based (C), and sector-based measurement (D); and corresponding histological section stained by Masson's trichrome (E). Note that necrotic tissue was recognized in the subendocardial layer in the LGE image (A) and in the section stained with Masson's trichrome (E) (as highlighted by arrows). The epicardium is traced in green and the endocardium is traced in red. Infarct area is delineated with yellow, and infarct length with blue lines. (F) Comparison of infarct size assessed with the 3 methods: area-based $(\bullet)$, length-based $(\boldsymbol{\bullet})$, and sector-based (threshold: mean + SD $[\bullet]$, mean $+2 \operatorname{SD}[\mathbf{\Delta}]$, and mean +3 SD $[\mathbf{\nabla}])$. $(\mathbf{G})$ Signal intensity distribution in sector-based analysis. Infarct sizes quantified by the 3 thresholds are $74.2 \%$ (mean + SD), $66.9 \%$ (mean $+2 \mathrm{SD}$ ), and $61.7 \%$ $($ mean $+3 \mathrm{SD})$.

consistent from 1 week to 5 weeks after the onset of MI despite a gradual increase in LV diameter.

In the case of subendomyocardial infarction as shown in Figure 4, sector-based measurement on an LGE image could give a similar infarct size to that obtained by length-based calculation on a histological section. The subendocardial infarct region was included in infarct myocardium by the length-based method in histology and sector-based approach with LGE, however, the same area was excluded in the other approaches. In this case, infarct transmurality has a considerable influence on the calculation of infarct size. In addition, the sector-based approach is less affected by observer bias effect because it needs only delineation of myocardium and calculation of the mean and SD from a remote region. In contrast, area-based and length-based measurements require manual demarcation of the infarcted region. Although the length-based method could improve the sensitivity of the measurement of the infarct extent compared to the area-based approach, manual delineation of infarct length is prone to subjective bias. Therefore, the sector-based approach would be a reliable alternative to the conventional estimation of infarct.

In this study, we compared area-based, length-based, and sector-based methods to assess infarct size on LGE CMR images of a mouse MI model. Our results indicate that the 3 measurement approaches were highly correlated in the assessment of infarct size. Although infarct sizes derived from the 3 
methods were significantly correlated with each other, there was no concordance between the measurement methods. Lichtenauer, et al used an angle-based approach to evaluate infarct size in a chronic MI model. ${ }^{10)}$ They observed that infarct size determined by area-based measurement was smaller than those obtained by both length-based and angle-based measurements (area-based: $14.8 \pm 1.3 \%$, length-based: $23.9 \pm 2.0 \%$, anglebased: $24.8 \pm 2.1 \%$ ) and suggested the angle-based measurement could be applied to cardiac MRI. Our sector-based approach with mean $+3 \mathrm{SD}$ threshold has a similar geometric basis to the angle-based method, and both are based on circumferential measurement from a central angle or arc length. Sector-based measurements showed a significant difference and trend toward reduced infarct sizes with increasing thresholds when more than 8 sectors were used for the calculation.

Although functional parameters such as LV ejection fraction and fractional shortening were not evaluated in this study, previous studies have shown significant correlations between cardiac functional parameters and infarct size assessed by areabased, length-based, and angle-based approaches. ${ }^{9,10,15}$

In conclusion, we demonstrated that the methods used for the histological calculation of infarct size could be applied to CMR investigation. Moreover, the sector-based approach is a simple and reliable method for measurement of infarct size on LGE CMR images and is less affected by morphological changes in myocardium.

\section{Disclosure}

Conflict of interest: There is no conflict of interest to report in this study.

\section{REFERENCES}

1. Eitel I, de Waha S, Wohrle J, et al. Comprehensive prognosis assessment by CMR imaging after ST-segment elevation myocardial infarction. J Am Coll Cardiol 2014; 64: 1217-26.

2. Risk stratification and survival after myocardial infarction. $\mathrm{N}$ Engl J Med 1983; 309: 331-6.
3. Miller TD, Christian TF, Hopfenspirger MR, Hodge DO, Gersh BJ, Gibbons RJ. Infarct size after acute myocardial infarction measured by quantitative tomographic $99 \mathrm{mTc}$ sestamibi imaging predicts subsequent mortality. Circulation 1995; 92: 334-41.

4. Thomson LE, Kim RJ, Judd RM. Magnetic resonance imaging for the assessment of myocardial viability. Journal of magnetic resonance imaging. J Magn Reson Imaging 2004; 19: 771-88. (Review)

5. Coolen BF, Paulis LE, Geelen T, Nicolay K, Strijkers GJ. Contrast-enhanced MRI of murine myocardial infarction - part II. NMR Biomed 2012; 25: 969-84. (Review)

6. Ota $\mathrm{S}$, Tanimoto $\mathrm{T}$, Orii $\mathrm{M}$, et al. Impact of low signal intensity assessed by cine magnetic resonance imaging on detection of poorly viable myocardium in patients with prior myocardial infarction. Int Heart J 2015; 56: $273-7$.

7. Rehwald WG, Fieno DS, Chen EL, Kim RJ, Judd RM. Myocardial magnetic resonance imaging contrast agent concentrations after reversible and irreversible ischemic injury. Circulation 2002; 105: 224-9.

8. Ota S, Tanimoto T, Hirata K, et al. Assessment of circumferential endocardial extent of myocardial edema and infarction in patients with reperfused acute myocardial infarction. Int Heart J 2014; 55 : 234-8.

9. Takagawa J, Zhang Y, Wong ML, et al. Myocardial infarct size measurement in the mouse chronic infarction model: comparison of area- and length-based approaches. J Appl Physiol 2007; 102: 2104-11

10. Lichtenauer M, Schreiber C, Jung C, et al. Myocardial infarct size measurement using geometric angle calculation. Eur J Clin Invest 2014; 44: 160-7.

11. Park SW, Lee SY, Park SJ, Lee SC, Gwon HC, Kim DK. Quantitative assessment of infarct size in vivo by myocardial contrast echocardiography in a murine acute myocardial infarction model. Int J Cardiol 2004; 97: 393-8.

12. Zhu B, Sun Y, Sievers RE, et al. Comparative effects of pretreatment with captopril and losartan on cardiovascular protection in a rat model of ischemia-reperfusion. J Am Coll Cardiol 2000; 35: 787-95.

13. Virag JI, Murry CE. Myofibroblast and endothelial cell proliferation during murine myocardial infarct repair. Am J Pathol 2003; 163: 2433-40.

14. Lutgens E, Daemen MJ, de Muinck ED, Debets J, Leenders P, Smits JF. Chronic myocardial infarction in the mouse: cardiac structural and functional changes. Cardiovascul Res 1999; 41: 586-93.

15. Pfeffer MA, Pfeffer JM, Fishbein MC, et al. Myocardial infarct size and ventricular function in rats. Circ Res 1979; 44: 503-12. 